J. Gynäkol. Endokrinol. AT 2020 · 30:101-110 https://doi.org/10.1007/s41974-020-00145-9 Online publiziert: 11. Juni 2020

(c) Der/die Autor(en) 2020

\section{Bettina Böttcher · Bettina Toth}

Klinik für gyn. Endokrinologie und Reproduktionsmedizin, Department Frauenheilkunde, Medizinische Universität Innsbruck, Innsbruck, Österreich

\title{
Update Fertilitätsprotektion bei Mädchen, Adoleszentinnen und Frauen
}

\section{Einleitung}

Die Fertilitätsprotektion bei Mädchen, Adoleszentinnen und Frauen hat sich in den letzten zwei Jahrzehnten rasant entwickelt. Aufgrund verbesserter Therapien bei onkologischen Patientinnen und einer dadurch verbesserten Überlebensrate rückt eine spätere Familienplanung zunehmend in den Fokus. Aber auch die Nachfrage nach fertilitätsprotektiven Maßnahmen bei nichtonkologischen Erkrankungen wie beispielsweise bei rheumatologischen Erkrankungen, genetischen Prädispositionen mit eingeschränkter Ovarreserve, die zu einer prämaturen Ovarialinsuffizienz (POI) führen, oder Endometriose ist steigend.

Chemotherapien, Strahlentherapien und Operationen können zur Schädigung der Ovarien bzw. zu einer eingeschränkten Ovarreserve führen. Eine irreversible Ovarschädigung hat eine prämature Ovarialinsuffizienz zur Folge, die einerseits zu einer Hormonmangelsituation, andererseits zu einer erschwerten bis unmöglichen Realisierung eines Kinderwunschs führen kann. Daher ist die Aufklärung und Beratung vor einer gonadotoxischen Therapie von enormer Bedeutung. Auch wenn dieser Aspekt zunehmend von den behandelnden Ärztinnen und Ärzten berücksichtigt wird, fühlen sich viele Frauen noch nicht ausreichend informiert [1]. Bei der individuellen Beratung über fertilitätsprotektive Methoden sollten im interdisziplinären Konsens Faktoren wie die Therapie, die Prognose, die jeweilige Ovarreserve, die Risiken und Kontraindikationen bei einer möglichen Schwangerschaft, aber auch persönliche Wünsche und Vorstellungen berücksichtigt werden.

Der Grad der ovariellen Schädigung durch eine gonadotoxische Therapie wird von mehreren Faktoren beeinflusst: Das Alter beider Erkrankung, die individuelle Ovarreserve vor der Therapie, Art und Dosis der Chemotherapie sowie Dosis und Zielbereich bei einer Strahlentherapie spielen wesentliche Rollen.

Etablierte fertilitätsprotektive Methoden sind die Kryokonservierung von befruchteten und unbefruchteten Eizellen, die Kryokonservierung von Ovarialgewebe mit späterer Transplantation, die medikamentöse Gabe von GnRH-Analoga und die Transposition der Ovarien aus dem kleinen Becken vor einer Strahlentherapie.

Im deutschsprachigen Raum wurde im Jahr 2006 das Netzwerk „FertiPROTEKT“ gegründet, das erfolgreich das Bewusstsein für Möglichkeiten der Fertilitätsprotektion bei Ärzten und Patientinnen stärkt, Empfehlungen publiziert und den wissenschaftlichen Austausch fördert. Eine Leitlinie über die Fertilitätsprotektion bei malignen Erkrankungen wurde von den deutschen, österreichischen und Schweizer Fachgesellschaften für Gynäkologie und Geburtshilfe kürzlich veröffentlicht [2].

Im Folgenden werden die etablierten Methoden der Fertilitätsprotektion bei Frauen, erweiterte Indikationen, die Problematik der Beratungssituation und der Kostenübernahme sowie Zukunftsperspektiven thematisiert.

\author{
Methoden der Fertilitäts- \\ protektion bei Frauen
}

\section{Ovarielle Stimulation und Kryokonservierung von Eizellen und/oder Embryonen}

Etablierte Techniken der künstlichen Befruchtung mit Kryokonservierung von befruchteten Eizellen/Embryonen können Patientinnen mit einem vorhandenen männlichen Partner angeboten werden. Die Kryokonservierung unbefruchteter Eizellen kommt primär für Frauen ohne Partner in Betracht. In der Praxis wird auch bei Vorhandensein eines Partners bei ausreichender Eizellanzahl häufig eine Kombination des Einfrierens von unbefruchteten und befruchteten Eizellen durchgeführt, um später beide Optionen in Anspruch nehmen zu können (sogenanntes „splitting“) und nicht von einem späteren Einverständnis des Partners mit einem Embryotransfer abhängig zu sein. Der Fortschritt bei der Technik der Vitrifikation, eines ultraschnellen Verfahrens des Einfrierens von Eizellen, hat die Ergebnisse der Eizellkryokonservierung deutlich verbessert [3], was beispielsweise auch beim sogenannten „social egg freezing“, dem Einfrieren von Eizellen, um die Familienplanung in eine spätere Lebensphase zu verschieben, genutzt wird. Dies ist - im Gegensatz zu Deutschland entsprechend dem Fortpflanzungsmedizingesetz von 2015 in Österreich jedoch nicht erlaubt.

Für die Eizellentnahme ist eine hormonelle ovarielle Stimulation erforderlich, die durchschnittlich 10-14 Tage 
in Anspruch nimmt. Mittlerweile wurden Stimulationsprotokolle etabliert, die einen Stimulationsstart unabhängig von der Zyklusphase erlauben. Im Hinblick auf die Anzahl gewonnener Eizellen zeigten sich im Vergleich zu klassischen Protokollen vergleichbare Ergebnisse $[4,5]$. Im Fall von östrogenabhängigen Tumoren wie dem hormonrezeptorpositiven Mammakarzinom werden Stimulationsprotokolle mit einem Aromataseinhibitor (z.B. Letrozol) empfohlen, um die Östrogenlevel niedrig $\mathrm{zu}$ halten. Gesamtüberleben und rezidivfreies Überleben unterschieden sich bei Frauen mit Mammakarzinom, die im Letrozolprotokoll stimuliert wurden, nicht von Frauen, die keine Stimulation hatten [6].

Entsprechend dem FertiPROTEKTNetzwerk werden durchschnittlich 5-9 Eizellen nach einer Stimulationsbehandlung gewonnen, die Rate an Komplikationen ist gering [7].

Hinsichtlich der onkologischen Therapieplanung muss beachtet werden, dass der Beginn einer Chemo- oder Strahlentherapie durch die hormonelle Stimulation und Eizellentnahme um wenigstens zehn Tage verzögert wird. Ob dies vertretbar ist, muss individuell und in interdisziplinärer Zusammenarbeit entschieden werden.

Eine In-vitro-Maturation von Eizellen kann eine weitere Option für Patientinnen mit einem hohen antralen FollikelCount und nicht ausreichend Zeit oder Kontraindikationen für eine ovarielle Stimulation sein. Leider sind die Erfolgsraten bei dieser Technik bisher nicht zufriedenstellend [8] und werden daher derzeit nur von wenigen Kinderwunschzentren angeboten.

\section{Kryokonservierung von Ovargewebe und Transplantation}

Die Kryokonservierung von Ovargewebe stellt eine weitere Option der Fertilitätsprotektion dar, deren Vorteil ist, dass eine Chemotherapie direkt am postoperativen Tag begonnen werden kann. Auch bei Adoleszentinnen wird diese Methode zunehmend angewandt. Selbstverständlich kann eine Kryokonservierung von Ovargewebe auch mit einer anschließenden ovariellen Stimulation mit Eizellentnah- me kombiniert werden, sofern genügend Zeit zur Verfügung steht.

Nach Transplantation von Ovargewebe wurden weltweit über 170 Kinder geboren, wobei bislang 130 Geburten publiziert wurden [9]. Lange wurde diese Technik als experimentell betrachtet, aber entsprechend der aktuellen deutschsprachigen AWMF-Leitlinie (Arbeitsgemeinschaft der Wissenschaftlichen Medizinischen Fachgesellschaften e.V.) zur Fertilitätsprotektion bei malignen Erkrankungen [2] gilt die Kryokonservierung von Ovargewebe als etablierte Methode.

Der Vorgang besteht aus der chirurgischen Entnahme des Ovargewebes, meist laparoskopisch, der Kryokonservierung mittels Slow-freezing-Verfahren und, nach einem rezidivfreien Intervall, der Transplantation des Ovargewebes. Die Entnahme kann zyklusunabhängig erfolgen und erfordert keine hormonelle Vorbehandlung.

Ein weiterer Vorteil der Ovarkryokonservierung ist neben der möglichen Wiederherstellung der Fertilität auch der Erhalt der endokrinen Funktion und die Verbesserung einer prämaturen Ovarialinsuffizienz.

Das Hauptrisiko bei der Kryokonservierung von Ovargewebe besteht in einer Rückverlagerung maligner Zellen bei der Transplantation. Dieses Risiko ist von der Art der Krebserkrankung abhängig und ist bei Patientinnen mit Leukämie, Neuroblastom und Burkitt-Lymphom besonders hoch [10]. Möglicherweise können verschiedene Verfahren dieses Risiko zukünftig minimieren, sodass nach entsprechender Aufklärung der Patientin dieses Verfahren dennoch angeboten werden kann. Es wird empfohlen, bestimmte Kriterien wie ein Alter unter 35 Jahren, eine relativ gute Ovarreserve, eine gute onkologische Prognose mit einer Überlebenszeit von mindestens fünf Jahren sowie ein Risiko für eine prämature Ovarialinsuffizienz von mindestens $50 \%$ bei der Indikationsstellung zu berücksichtigen [9].

Zum Zeitpunkt der Transplantation wird das fragmentierte Ovargewebe entweder orthotop auf das Ovar oder in eine Peritonealtasche bzw. heterotop außerhalb des kleinen Beckens transplan- tiert. Sofern die Eileiter durchgängig sind und das Ovargewebe seine Funktion wieder aufgenommen hat, ist nach einer orthotopen Transplantation eine natürliche Konzeption möglich. Die ovarielle Aktivität beginnt durchschnittlich vier Monate nach der Transplantation und hält für etwa fünf Jahre an. Die Prozedur kann bei Nachlassen der ovariellen Funktion wiederholt werden, da jeweils nur Anteile des eingefrorenen Gewebes transplantiert werden [11].

Wenn nicht ausreichend Zeit für eine hormonelle Stimulation ist oder die Art des Karzinoms es nicht zulässt, kann eine In-vitro-Maturation von unreifen Eizellen mit der Kryokonservierung von Ovargewebe kombiniert werden [12]. Die Eizellen können entweder durch eine Punktion der sichtbaren Antralfollikel oder während der Aufarbeitung des Ovargewebes gewonnen werden [13]. Diese Techniken gelten noch als experimentell.

\section{Transposition der Ovarien}

Strahlentherapeutische Methoden haben sich in den letzten Jahren deutlich verbessert, sodass es mittlerweile möglich ist, die Dosis und den lokalen Effekt auf die Ovarien zu ermitteln [14]. Man geht davon aus, dass eine Gesamtdosis von 16 Gy für die Ovarien irreversibel toxisch wirkt [15]. Vor einer geplanten Strahlentherapie des Beckens können die Eierstöcke uni- oder bilateral aus dem Strahlenfeld heraus an der Beckenwand fixiert werden. Diese laparoskopisch durchgeführte Transposition kann die mögliche Schädigung der Ovarien durch die Strahlentherapie verringern [16] und auch bei Mädchen durchgeführt werden [15]. Risiken beinhalten neben den üblichen Operationsrisiken selten postoperative Ovarialzysten [15]. Die Datenlage im Hinblick auf Erfolgschancen ist limitiert. Die Entscheidung sollte unter Berücksichtigung der Erkrankung, der Prognose, der Gesamtstrahlendosis und der eventuellen Kombination mit einer Chemotherapie getroffen werden. Auch sollte beachtet werden, dass der Uterus durch eine Strahlentherapie geschädigt werden kann, wodurch eine Schwangerschaft entweder nicht möglich oder der 
Verlauf durch Fehl- und Frühgeburten deutlich erschwert sein kann.

\section{Medikamentöser Ansatz mit GnRH-Analoga}

Die Gabe von GnRH-Analoga zur Minimierung der gonadalen Toxizität wird weiterhin kontrovers diskutiert [2, 17]. Eine Vielzahl von Studien in verschiedenen Kohorten wurde mittlerweile mit widersprüchlichen Ergebnissen publiziert. Outcomeparameter für die Wirksamkeit von GnRH-Analoga sind nicht standardisiert und reichen von Werten des Anti-Müller-Hormons und/oder des follikelstimulierenden Hormons (FSH) bis zu Amenorrhö und Schwangerschaftsraten [18]. Beispielsweise zeigte eine prospektive, randomisierte Studie bei Lymphompatientinnen keinen „benefit" der GnRH-Analoga [19], wohingegen in zwei prospektiven Studien bei Frauen mit Mammakarzinom ermutigende Ergebnisse im Hinblick auf höhere Schwangerschaftsraten und die Wiederherstellung der ovariellen Funktion bei Frauen, die GnRH-Analoga erhalten hatten, publiziert wurden [20, 21].

Eine aktuelle Metaanalyse zeigte, dass GnRH-Analoga im Hinblick auf das Wiedereintreten der Menstruation und Ovulation und auf eine prämature Ovarialinsuffizienz effizient zu sein scheinen, jedoch die Datenlage hinsichtlich Schwangerschaftsraten und einer Fertilitätsprotektion weiterhin nicht eindeutig ist [22]. Daher sollte man sich nicht auf GnRHAnaloga als alleinige Maßnahme verlassen, sondern die Kombination mit anderen fertilitätsprotektiven Methoden anbieten [2].

\section{Benigne Indikationen}

Die Indikationen, bei denen fertilitätsprotektive Maßnahmen angewandt werden, beinhalten nicht nur onkologische Erkrankungen. Weitere Indikationen sind rheumatische Erkrankungen wie ein systemischer Lupus erythematodes, bei denen eine zytotoxische Therapie erforderlich ist, und genetische Prädispositionen wie das Turner-Syndrom, die mit einer reduzierten ovariellen Reserve einhergehen können.

Selbstverständlich ist auch bei diesen Indikationen eine interdisziplinäre Zusammenarbeit zu empfehlen. Auch sollten Kontraindikationen gegen eine Schwangerschaft abgeklärt werden.

Des Weiteren stellen wiederholte chirurgische Eingriffe bei Patientinnen mit Endometriose ein Risiko für eine Verringerung der Ovarialreserve dar. Daher kann den Patientinnen je nach individueller Situation eine ovarielle Stimulation und Kryokonservierung von Oozyten und/oder Embryonen angeboten werden.

Zunehmend nachgefragt werden fertilitätsprotektive Maßnahmen durch Trans $^{\star}$ personen. Idealerweise können vor Beginn einer gegengeschlechtlichen

Hier steht eine Anzeige. 
Hormontherapie Eizellen oder Spermien kryokonserviert werden.

\section{Kosten}

Die Kostenübernahme für fertilitätsprotektive Maßnahmen in Österreich ist leider noch nicht abschließend geregelt und unterscheidet sich sowohl zwischen den Ländern als auch zwischen einzelnen Instituten und Kliniken. In Österreich können die Kosten für eine ovarielle Stimulation, sofern ein Partner vorhanden ist und die üblichen Bedingungen erfüllt sind, teilweise vom IVF-Fonds übernommen werden. Die Kosten für eine Ovarkryokonservierung sind im Hinblick auf die Kosten der Operation und der Aufarbeitung des Gewebes sehr unterschiedlich. Hinzu kommen jährliche Lagerungskosten für Oozyten, Embryonen und Ovarialgewebe. Teilweise werden Anträge bei der Krankenkasse für die Kostenübernahme von GnRH-Analoga bewilligt.

Im Gegensatz zu Österreich wurde in Deutschland im letzten Jahr (2019) ein Gesetzesentwurf verabschiedet, bei dem der Leistungsanspruch für eine künstliche Befruchtung um die Kryokonservierung von Ovarialgeweben bei medizinischen Indikationen wie Krebserkrankungen oder rheumatischen Erkrankungen erweitert wurde. Allerdings ist die detaillierte Umsetzung durch den Gemeinsamen Bundesausschuss der Krankenkassen derzeit ausständig. Die Kosten für eine ovarielle Stimulation können bei verheirateten Paaren und Erfüllung der übrigen Bedingungen von den Krankenkassen übernommen werden. Lagerungskosten müssen selbst getragen werden.

Es wäre wünschenswert, wenn auch in Österreich eine Unterstützung der fertilitätsprotektiven Maßnahmen in den Leistungskatalog der Krankenkassen aufgenommen wird, damit nicht finanzielle Probleme dafür ursächlich sind, dass eine Patientin diese Maßnahmen nicht in Anspruch nehmen und ihren Kinderwunsch realisieren kann.

J. Gynäkol. Endokrinol. AT 2020 · 30:101-110 https://doi.org/10.1007/s41974-020-00145-9

(c) Der/die Autor(en) 2020

\section{B. Böttcher · B. Toth \\ Update Fertilitätsprotektion bei Mädchen, Adoleszentinnen und Frauen}

\section{Zusammenfassung}

Die Fertilitätsprotektion, insbesondere bei Frauen, hat sich in den letzten Jahren rasant weiterentwickelt. Aufgrund steigender Heilungsraten bei Krebserkrankungen bei jungen Frauen rückt die Bedeutung einer späteren Familienplanung zunehmend in den Fokus. Dennoch erhält nicht jede Patientin vor einer gonadotoxischen Chemo- und/oder Strahlentherapie eine adäquate Beratung über die verfügbaren fertilitätsprotektiven Methoden, die die individuelle Situation, Erkrankung, geplante Therapie, Prognose und persönlichen Wünsche berücksichtigt. Der Entscheidungsprozess ist weiterhin eine Herausforderung für alle beteiligten Disziplinen wie die Onkologie, Chirurgie, Urologie, Gynäkologie, Reproduktionsmedizin, Pädiatrie und Strahlentherapie. Aktuelle Methoden der Fertilitätsprotektion bei Frauen beinhalten die Kryokonservierung von befruchteten und/oder unbefruchteten Eizellen, die Kryokonservierung von Ovarialgewebe, die Verlagerung der Ovarien aus dem kleinen Becken vor einer Strahlentherapie und die medikamentöse Behandlung mittels Gonadotropin-ReleasingHormon(GnRH)-Analoga. Verschiedene Forschungsgruppen beschäftigen sich aktuell mit der Optimierung des Überlebens der Follikel im entnommenen Ovarialgewebe, der Minimierung des Risikos, maligne Zellen zu transplantieren, mit Möglichkeiten, das Ovar besser vor chemotherapeutischen Schäden zu schützen, und der Entwicklung eines sogenannten „künstlichen Ovars". Aktuelle Methoden und Zukunftsperspektiven der Fertilitätsprotektion bei Frauen werden in diesem Artikel dargestellt.

\section{Schlüsselwörter}

Hormonelle Stimulation - Ovarkryokonservierung · GnRH-Analoga · Künstliches Ovar · Invitro-Maturation

\section{Update on Fertility Preservation in Girls, Adolescents, and Women}

\section{Abstract}

The demand for fertility preservation in women has increased over the past few years and should be an integral part of treatment for young women with planned gonadotoxic therapy. Thanks to improved survival rates, future family planning is attracting more attention. Nevertheless, not every woman receives adequate counselling about options for fertility preservation before starting gonadotoxic therapy. The decision-making process remains a challenge for all disciplines involved in therapy planning such as surgery, oncology, urology, gynecology, reproductive medicine, pediatrics, and radiotherapy. Available options for fertility preservation in women include: cryopreservation of oocytes, embryos, and ovarian tissue; transposition of ovaries before radiotherapy; and medical treatment with gonadotropin- releasing hormone $(\mathrm{GnRH})$ analogues. These techniques have been established and improved but further improvements are necessary to enable these women to start family planning in the future. Various research groups aim to improve the survival of follicles in the ovarian graft and to minimize the risk of re-seeding malignant cells after ovarian tissue transplantation, and are working on the development of an "artificial ovary" as well as the protection of the ovary by medical agents. This review summarizes the currently available techniques for fertility preservation in women and discusses future perspectives.

\section{Keywords}

Hormonal stimulation - Cryopreservation of ovarian tissue $\cdot \mathrm{GnRH}$ analogues · Artificial ovary $\cdot$ In vitro maturation

\section{Zukunftsperspektiven}

\section{Gonadoprotektive Substanzen}

Der Mechanismus gonadoprotektiver Substanzen beruht auf der Theorie, dass der chemotherapieinduzierte Verlust an Follikeln durch Apoptose von großen Follikeln und gleichzeitig durch die Aktivierung von ruhenden Follikeln gekennzeichnet ist. Daher liegt der Fokus aktueller Forschung auf Substanzen mit antiapoptotischen Charakteristika und der Fähigkeit, die Follikelaktivie- 
rung zu verhindern: Sphingosin-1-phosphat (S1P) blockiert die Apoptose, die durch bestimmte Zytostatika induziert wird, und schützt bestrahlte Follikel im Tierversuch durch die Inhibierung eines ceramidvermittelten Signaltransduktionswegs [23-25]. Bisher kann die Substanz allerdings nicht systemisch verabreicht werden und hat eine kurze Halbwertszeit, wodurch die Einsatzmöglichkeiten limitiert sind [26].

Imatinib ist ein c-Abl-TyrosinkinaseInhibitor, der die Schädigung der Ovarfollikel bei Mäusen bei gleichzeitiger Gabe von Cisplatin verringern konnte [27].

Es konnte weiterhin gezeigt werden, dass Tamoxifen, ein selektiver Östrogenrezeptormodulator, den Follikelverlust und die Eizellfragmentierung unter Koadministration von Doxorubicin und Cyclophosphamid im Tierversuch mit Ratten vermindern konnte [28]. Des Weiteren konnte ebenso bei Ratten ein Effekt von Tamoxifen auf einen strahleninduzierten Follikelverlust demonstriert werden, der auf einer Stabilisierung des Anti-Müller-Hormons (AMH), Erhöhung der Insulin-like-growth-factor-1Level (IGF-1) und einem Entgegenwirken einer durch oxidativen Stress induzierten Apoptose zu beruhen scheint [29].

Der Immunmodulator AS101 verhindert die Follikelaktivierung über eine Inhibition des PI3K/PTEN/Akt-Signaltransduktionswegs und schützt das Ovar hiermit vor dem „Burnout“-Effekt der Chemotherapie. AS101 wurde Mäusen gleichzeitig mit Cyclophosphamid verabreicht, wobei sich eine Hemmung der Follikelaktivierung und nachfolgend eine höhere Ovarialreserve zeigte. Interessanterweise war bei den Mäusen der zytotoxische Effekt von Cyclophosphamid auf Mammakarzinomzellen erhöht [30]. Ein weiterer Ansatz könnte die Apoptoseinhibition durch Ceramid-1Phosphat sein, wie im Mausversuch mit Cyclophosphamid gezeigt werden konnte [31].

\section{Erhöhung des follikulären Überle- bens nach der Ovartransplantation}

Die Kryokonservierung von Ovargewebe mit späterer Transplantation ist zu einer vielversprechenden Methode der Fertilitätsprotektion geworden, die bislang $\mathrm{zu}$ über 170 Lebendgeburten weltweit geführt hat [9]. Allerdings wurde nach der Transplantation ein massiver Verlust an Follikeln beobachtet, der möglicherweise durch eine langsame Vaskularisation des Gewebes mit nachfolgender Ischämie zu erklären ist [32]. Daher untersuchen verschiedene Forschungsgruppen Substanzen, die die Vaskularisation durch Angiogenese und antiapoptotische Mechanismen verbessern können. Der Einfluss folgender Substanzen auf kryokonserviertes Ovargewebe mit teilweise vielsprechenden Ergebnissen wurde bisher experimentell untersucht: Eine Kombination aus Melatonin, Vitamin E, Hyaluron und „vascular endothelial growth factor $A$ “ (VEGF-A) erhöhte die Neovaskularisation und reduzierte apoptotische Vorgänge [32]. Des Weiteren wurden experimentelle Studiendesigns mit Erythropoetin, VEGF111, Fibroblastenwachstumsfaktoren, Simvastatin und eine subkutane Pumpe mit S1P entwickelt [26]. Zwei Schwangerschaften und eine Lebendgeburt wurden nach Transplantation von Ovarialgewebe mit einer speziellen extrazellulären Matrix, die das Überleben von Primordialfollikeln förderte, publiziert [33].

\section{Risikoreduktion der Rückübertra- gung maligner Zellen}

\section{In-vitro-Wachstum und Maturation von Primordialfollikeln}

Die Transplantation des kryokonservierten Ovargewebes birgt das Risiko, maligne Zellen zu übertragen. Verfahren wie das sogenannte ,in vitro growth“, das Wachstum unreifer Eizellen in Kultur, und die In-vitro-Maturation von Eizellen können dieses Risiko verringern. Das Ziel ist die Vollendung des gesamten Follikelwachstums in vitro, um befruchtungsfähige Eizellen zu erhalten. Bei Mäusen sind nach Anwendung dieser Methode Lebendgeburten beschrieben [34, 35]. Für Ovargewebe und Follikel wurden verschiedene Kultursysteme entwickelt [35-39], wobei jedes Stadium des Follikelwachstums eigene Bedingungen erfordert, wodurch die Entwicklung eines einzigen Kultursystems eine Herausfor- derung ist. Des Weiteren müssen die Follikelmorphologie und kritische ZellZell-Interaktionen erhalten bleiben [40]. Die ideale Matrix für das Follikelwachstum wurde bisher noch nicht gefunden. Es wurde versucht, den Kultursystemen verschiedene Wachstumsfaktoren hinzuzufügen, aber das Follikelwachstum arretierte oft im frühen Sekundärstadium [26]. Ein zweistufiges Kultursystem, das von Telfer et al. [38] entwickelt wurde, initiiert im ersten Schritt das Wachstum von Primordialfollikeln und im zweiten Schritt die Entwicklung von Sekundärfollikeln nach Isolation in spezieller Kultur bis hin zu frühen Antralfollikeln. In einem mehrstufigen Kultursystem gelang das Wachstum bis zu MetaphaseII-Eizellen [41]. Die Entwicklung einer dreidimensionalen Alginat-HydrogelMatrix ermöglichte den Erhalt der morphologischen Struktur der Follikel. Nach der Isolation von Sekundärfollikeln wurden diese bis zu 40 Tagen kultiviert und anschließend eine In-vitro-Maturation der Eizellen durchgeführt [42].

Weitere Forschungsergebnisse zeigen, dass die Follikelaktivierung durch die Unterbrechung des Hippo-Signaltransduktionswegs und die Inhibition des PI3K-PTEN-Akt-Signaltransduktionswegs bei präpuberalen Mädchen und Frauen mit einer prämaturen Ovarialinsuffizienz erreicht werden kann [43]. Eine weitere Hypothese besteht darin, dass der Verlust von Primordialfollikeln nach Ovartransplantation mit einer Unterbrechung des PI3K/Akt-Signaltransduktionswegs zusammenhängen könnte [44].

Diese zwei Signaltransduktionswege scheinen daher eine wesentliche Rolle bei der Aktivierung von Primordialfollikeln zu spielen: Der PI3KPTEN-Akt-Weg ist an der Regulation des Ruhezustands der Follikel beteiligt, indem er für hohe Level von Phosphatidylinositol-4,5-bisphosphat (PIP2) und gleichzeitig zu niedrigeren Leveln von Phosphatidylinositol-3,4,5triphosphat (PIP3) sorgt. Die Inkubation von kryokonserviertem Ovargewebe mit Substanzen, die die PIP3-Werte erhöhen, hat bisher zu drei klinischen Schwangerschaften nach Transplantation geführt [45-47]. 


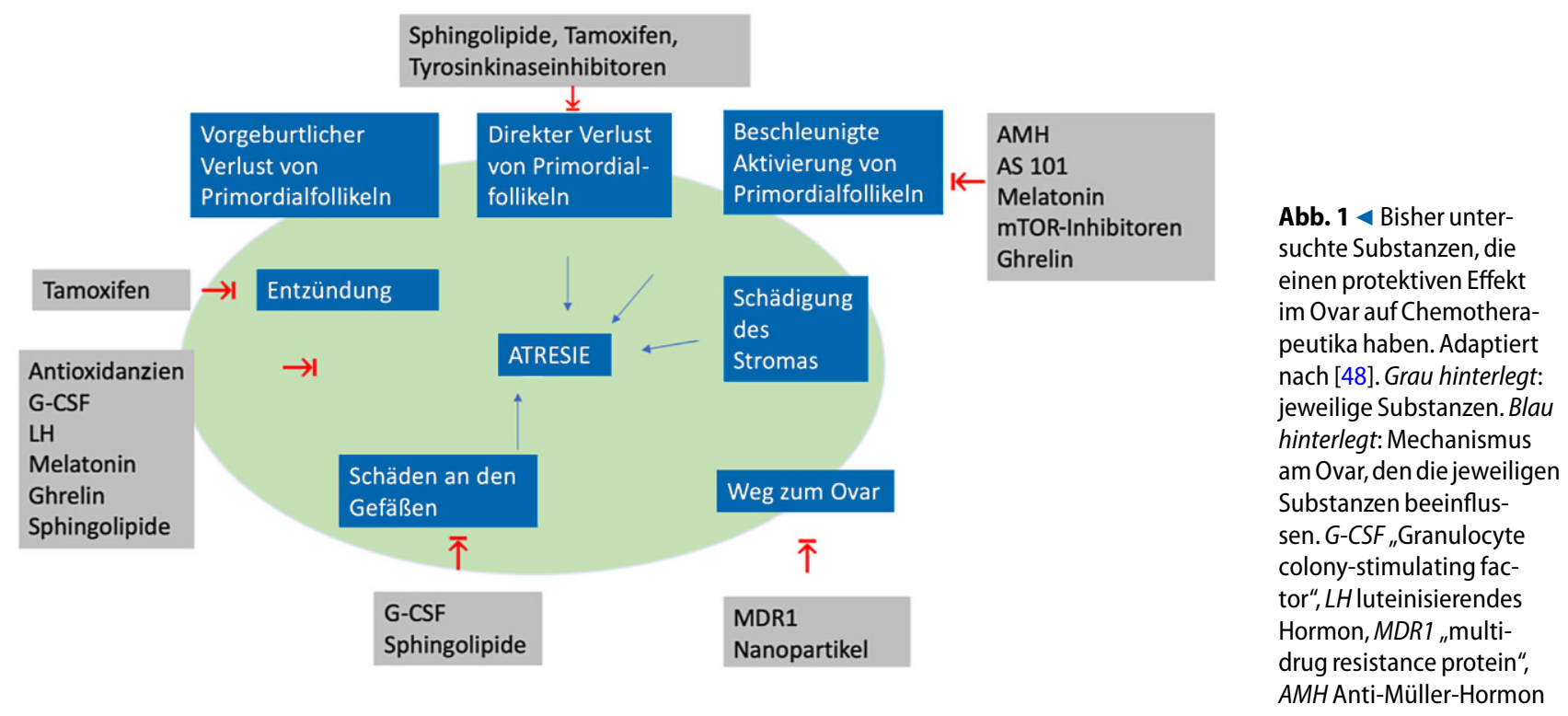

Der Hippo-Signaltransduktionsweg ist in Vorgänge der Zellproliferation, Apoptose und Follikelentwicklung involviert [13, 43]. Bei Unterbrechung dieses Wegs, wie es bei Schnittprozeduren des Ovars erfolgt, werden Zellwachstum und Proliferation beschleunigt. Diese Fragmentierung führt zur Entwicklung von Präantralfollikeln [43] und, nach Hinzugabe von Akt-Stimulatoren zum Kultursystem, zum Wachstum von Primordialfollikeln bei Patientinnen mit einer prämaturen Ovarialinsuffizienz [45].

Zusammenfassend sind dies vielversprechende Ergebnisse, wobei noch bestätigt werden muss, dass die in vitro gereiften Eizellen auch in der Lage sind, die Reifungsprozesse zu beenden, und dass Vorgänge des sogenannten "genomic imprinting" stattfinden [40], sodass eine klinische Anwendung ohne Risiken möglich sein wird.

Einen Überblick über mögliche Substanzen, die einen potenziellen gonadoprotektiven Effekt über unterschiedliche Mechanismen haben, bietet 0 Abb. 1 [48].

\section{Das „künstliche Ovar“}

Die Entwicklung eines „künstlichen Ovars" ist eine weitere mögliche Alternative, um reife Eizellen zu gewinnen und das Risiko für die Übertragung maligner Zellen im Rahmen einer Ovartransplantation $\mathrm{zu}$ verringern. Hierfür werden Primordialfollikel isoliert und in einer dreidimensionalen Matrix mit Alginat, Fibrin, Gelatine oder Polyethylenglykol fixiert [49-51]. Diese Matrix wird dann transplantiert. Das künstliche Ovar benötigt Stromazellen, die noch immer ein Restrisiko für maligne Zellen bergen: Hierfür könnten möglicherweise frische humane Knochenmarkzellen eine Alternative darstellen [52].

\section{Stammzellen}

Die Verwendung embryonaler oder induzierter pluripotenter Stammzellen könnte eine weitere Option sein, Gameten zu gewinnen. Möglicherweise sind auch Keimbahnstammzellen oder Vorläuferzellen im Ovar vorhanden. Aber auch hier besteht die Frage, ob diese Zellen auch für komplexe Mechanismen des "genomic imprinting“ und epigenetischer Abläufe geeignet sind [9, 53, 54].

\section{Allogenes Transplant oder Xenotransplantation}

Die Transplantation eines Ovars einer anderen Frau wäre, unter Berücksichtigung der Einschränkungen und Risiken, vergleichbar mit der Transplantation anderer Organe und eine Möglichkeit, die ovarielle Funktion bei einer prämaturen Ovarialinsuffizienz zurückzugewinnen.
Diese Prozedur wurde bei zwei Schwestern bereits durchgeführt und führte zur ersten publizierten Lebendgeburt nach allogener Ovartransplantation [55].

Auch die Transplantation von kryokonserviertem Gewebe in eine andere Spezies (z.B. immundefiziente Maus), um in dieser Eizellen zu maturieren und nach Befruchtung den Frauen zu transferieren, könnte eine Option zur Reduktion des Risikos der Übertragung maligner Zellen sein [56].

\section{Zusammenfassung}

Die Fertilitätsprotektion stellt ein relevantes Thema für Frauen vor einer gonadotoxischen Therapie, mit einer genetischen Prädisposition für eine prämature Ovarialinsuffizienz oder benignen Erkrankungen, die die ovarielle Reserve einschränken können, dar. Etablierte Techniken sind Eizell- und Embryonenkryokonservierung, die Kryokonservierung von Ovargewebe, die Transposition des Ovars vor einer Strahlentherapie und die Gabe von GnRH-Analoga. Die Beratung dieser Frauen ist eine interdisziplinäre Herausforderung [57], bei der viele Faktoren wie Zeit für die Beratung und Zeit bis zum Therapiestart, Eröffnung eines zusätzlichen Themas neben der geplanten onkologischen Therapie oder mangelndes Wissen eine Rolle spielen (• Abb. 2). Dies darf jedoch 


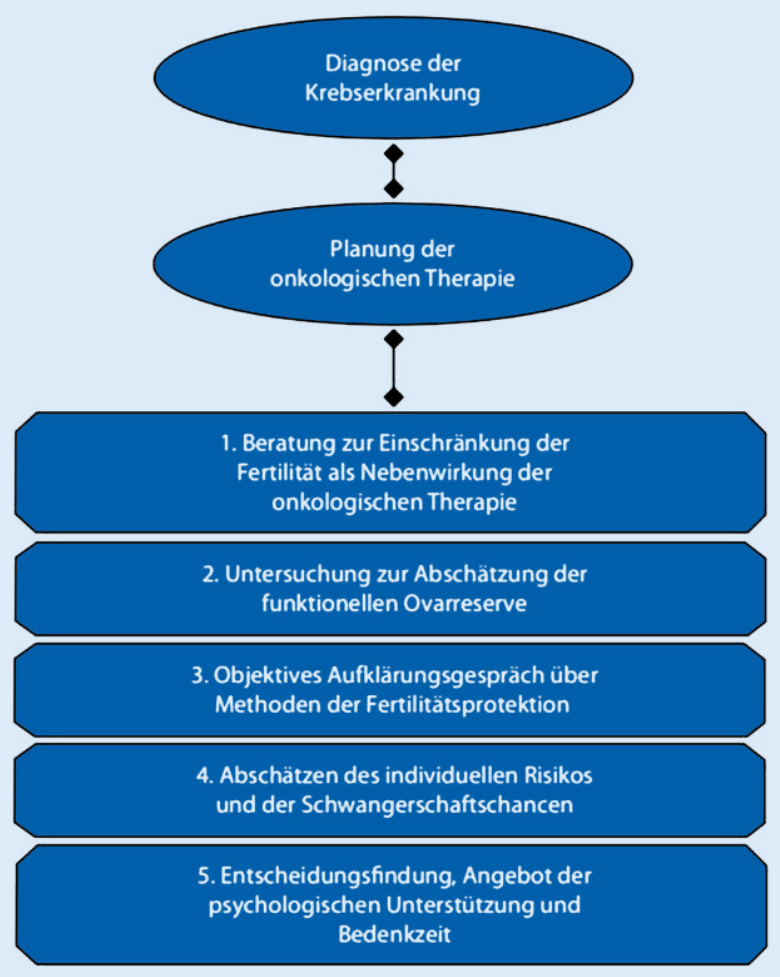

Abb. $2 \triangleleft$ Beratungskonzept zur Fertilitätsprotektion bei Krebspatientinnen. Nachdruck mit Genehmigung aus [59] nicht dazu führen, dass den Patientinnen Informationen über die aktuellen Techniken des Fertilitätserhalts vorenthalten werden. Frauen und ihren Partner/innen sollte eine autonome Entscheidung im Hinblick auf ihre spätere Familienplanung ermöglicht werden [58].

\section{Fazit für die Praxis}

- Frauen vor einer gonadotoxischen Therapie sollten über die Möglichkeiten der Fertilitätsprotektion informiert werden, wobei die Beratung interdisziplinär erfolgen soll.

- Auch bei benignen Erkrankungen wie Autoimmunerkrankungen und genetischen Prädispositionen, die mit einer reduzierten ovariellen Reserve einhergehen, steigt die Nachfrage nach fertilitätsprotektiven Maßnahmen.

- Etablierte Methoden sind Eizell- und Embryonenkryokonservierung, die Kryokonservierung von Ovargewebe, die Transposition des Ovars vor einer

Hier steht eine Anzeige.

\section{Springer}




\section{Strahlentherapie und die Gabe von GnRH-Analoga. \\ - Die Kostenübernahme durch Kran- kenkassen oder andere Institutionen in Österreich sollte gewährleistet werden, um möglichst vielen Frauen diese Optionen zu ermöglichen. \\ - Aktuelle Forschungsgebiete liegen in der Entwicklung eines „künstlichen Ovars", der Erhöhung der follikulären Überlebensrate nach einer Transplan- tation und der Reduktion des Risikos, maligne Zellen zu übertragen.}

\section{Korrespondenzadresse

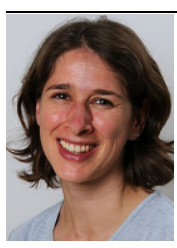 \\ Dr. med. Bettina Böttcher, \\ MA \\ Klinik für gyn. Endokrinologie und Reproduktionsmedizin, Department Frauenheilkun- de, Medizinische Universität Innsbruck \\ Anichstraße 35, 6020 Inns- \\ bruck, Österreich \\ bettina.boettcher@ \\ i-med.ac.at}

Funding. Open access funding provided by University of Innsbruck and Medical University of Innsbruck.

\section{Einhaltung ethischer Richtlinien}

Interessenkonflikt. B. Böttcher und B. Toth geben an, dass kein Interessenkonflikt besteht.

Für diesen Beitrag wurden von den Autoren keine Studien an Menschen oder Tieren durchgeführt. Für die aufgeführten Studien gelten die jeweils dort angegebenen ethischen Richtlinien.

Open Access. Dieser Artikel wird unter der Creative Commons Namensnennung 4.0 International Lizenz veröffentlicht, welche die Nutzung, Vervielfältigung Bearbeitung, Verbreitung und Wiedergabe in jeglichem Medium und Format erlaubt, sofern Sie den/die ursprünglichen Autor(en) und die Quelle ordnungsgemäß nennen, einen Link zur Creative Commons Lizenz beifügen und angeben, ob Änderungen vorgenommen wurden.

Die in diesem Artikel enthaltenen Bilder und sonstiges Drittmaterial unterliegen ebenfalls der genannten Creative Commons Lizenz, sofern sich aus der Abbildungslegende nichts anderes ergibt. Sofern das betreffende Material nicht unter der genannten Creative Commons Lizenz steht und die betreffende Handlung nicht nach gesetzlichen Vorschriften erlaubt ist, ist für die oben aufgeführten Weiterverwendungen des Materials die Einwilligung des jeweiligen Rechteinhabers einzuholen.
Weitere Details zur Lizenz entnehmen Sie bitte der Lizenzinformation auf http://creativecommons.org/ licenses/by/4.0/deed.de.

\section{Literatur}

1. Goossens J, Delbaere I, Van Lancker A, Beeckman D, Verhaeghe S, Van Hecke A (2014) Cancer patients' and professional caregivers' needs, preferences and factors associated with receiving and providing fertility-related information: a mixedmethods systematic review. Int J Nurs Stud 51(2):300-319

2. Dittrich $R$, Kliesch $S$, Schüring $A$, Balcerek $M$, Baston-Büst DM, Beck R et al (2018) Fertility preservation for patients with malignant disease. Guideline of the DGGG, DGU and DGRM (S2kLevel, AWMF Registry No. 015/082, November 2017)-Recommendations and statements for girls and women. Geburtshilfe Frauenheilkd 78(6):567-584

3. Dolmans M-M, Manavella DD (2019) Recent advances in fertility preservation. J Obstet Gynaecol Res 45(2):266-279

4. Cakmak H, Katz A, Cedars MI, Rosen MP (2013) Effective method for emergency fertility preservation: random-start controlled ovarian stimulation Fertil Steril 100(6):1673-1680

5. von Wolff M, Capp E, Jauckus J, Strowitzki T, Germeyer A (2016) FertiPROTEKT study group. Timing of ovarian stimulation in patients prior to gonadotoxic therapy: an analysis of 684 stimulations. Eur J Obstet Gynecol Reprod Biol 199:146-149

6. Azim AA, Costantini-Ferrando M, Oktay K (2008) Safety of fertility preservation by ovarian stimulation with letrozole and gonadotropins in patients with breast cancer: a prospective controlled study. JClin Oncol 26(16):2630-2635

7. Lawrenz B, Jauckus J, Kupka M, Strowitzki T, von Wolff M (2010) Efficacy and safety of ovarian stimulation before chemotherapy in 205 cases. Fertil Steril 94(7):2871-2873

8. Cao Y-X, Chian R-C (2009) Fertility preservation with immature and in vitro matured oocytes. Semin Reprod Med 27(6):456-464

9. Donnez J, Dolmans M-M (2017) Fertility preservation in women. NEngl J Med 377(17):1657-1665

10. Dolmans M-M, Masciangelo R (2018) Risk of transplanting malignant cells in cryopreserved ovarian tissue. Minerva Ginecol 70(4):436-443

11. Donnez J, Dolmans M-M, Pellicer A, Diaz-Garcia C, Sanchez Serrano M, Schmidt KT et al (2013) Restoration of ovarian activity and pregnancy after transplantation of cryopreserved ovarian tissue: a review of 60 cases of reimplantation. Fertil Steril 99(6):1503-1513

12. Chian R-C, Uzelac PS, Nargund G (2013) In vitro maturation of human immatureoocytes for fertility preservation. Fertil Steril 99(5):1173-1181

13. Winkler-CrepazK, Böttcher B, Toth B, Wildt L, HoferTollinger $S$ (2017) What is new in 2017? Update on fertility preservation in cancer patients. Minerva Endocrinol 42(4):331-339

14. Wallace WHB, Thomson AB, Saran F, Kelsey TW (2005) Predicting age of ovarian failure after radiation to a field that includes the ovaries. Int J Radiat Oncol Biol Phys 62(3):738-744

15. IrtanS, Orbach D, HelfreS, SarnackiS (2013) Ovarian transposition in prepubescent and adolescent girls with cancer. Lancet Oncol 14(13):e601-e608
16. Hoekman EJ, Broeders EABJ, Louwe LA, Nout RA, Jansen FW, de Kroon CD (2019) Ovarian function after ovarian transposition and additional pelvic radiotherapy: A systematic review. Eur JSurg Oncol 45(8):1328-1340

17. Practice Committee of the American Society for Reproductive Medicine (2019) Fertility preservation in patients undergoing gonadotoxic therapy or gonadectomy: a committee opinion. Fertil Steril 112(6):1022-1033

18. Hickman LC, Llarena NC, Valentine LN, Liu X, Falcone $T$ (2018) Preservation of gonadal function in womenundergoing chemotherapy:asystematic review and meta-analysis of the potential role for gonadotropin-releasing hormone agonists. J Assist Reprod Genet 35(4):571-581

19. Demeestere I, Brice P, Peccatori FA, Kentos A, Dupuis J, Zachee P et al (2016) No evidence for the benefit of gonadotropin-releasing hormone agonist in preserving ovarian function and fertility in lymphoma survivors treated with chemotherapy: finallong-term report of a prospective randomized trial.J Clin Oncol 34(22):2568-2574

20. Moore HCF, Unger JM, Phillips K-A, Boyle F, Hitre E, Porter D et al (2015) Goserelin for ovarian protection during breast-cancer adjuvant chemotherapy. N Engl J Med 372(10):923-932

21. Lambertini M, Boni L, Michelotti A, Gamucci T, Scotto T, Gori S et al (2015) Ovarian suppression with triptorelin during adjuvant breast cancer chemotherapy and long-term ovarian function, pregnancies, and disease-free survival: a randomized clinical trial. JAMA 314(24):2632-2640

22. Chen H, Xiao L, Li J, Cui L, Huang W (2019) Adjuvant gonadotropin-releasing hormone analogues for the prevention of chemotherapy-induced premature ovarian failure in premenopausal women. Cochrane Database Syst Rev 3(1):CD8018

23. Li F, Turan V, Lierman $S$, Cuvelier $C$, De Sutter $P$, Oktay K (2014) Sphingosine-1-phosphate prevents chemotherapy-induced human primordial follicle death. Hum Reprod 29(1):107-113

24. Zelinski MB, Murphy MK, Lawson MS, Jurisicova A, Pau KYF, Toscano NP et al (2011) In vivo delivery of FTY720 prevents radiation-induced ovarian failure and infertility in adult female nonhuman primates. Fertil Steril 95(4):1440-1445.e7

25. Kaya $H$, Desdicioglu $R$, Sezik M, Ulukaya $E_{\text {, }}$ Ozkaya O, Yilmaztepe A et al (2008) Does sphingosine-1-phosphate have a protective effect on cyclophosphamide- and irradiation-induced ovarian damage in the rat model? Fertil Steril 89(3):732-735

26. Fisch B, Abir R (2018) Female fertility preservation: past, present and future. Reproduction 156(1):F11-F27

27. Gonfloni S, Di Tella L, Caldarola S, Cannata SM, Klinger FG, Di Bartolomeo C et al (2009) Inhibition of the c-Abl-TAp63 pathway protects mouse oocytes from chemotherapy-induced death. Nat Med 15(10):1179-1185

28. Ting AY, Petroff BK (2010) Tamoxifen decreases ovarian follicular loss from experimental toxicant DMBA and chemotherapy agents cyclophosphamide and doxorubicin in the rat. J Assist Reprod Genet 27(11):591-597

29. Mahran YF, El-DemerdashE, Nada AS, Ali AA, AbdelNaim AB (2013) Insights into the protective mechanisms of tamoxifen in radiotherapy-induced ovarian follicular loss: impact on insulin-like growth factor 1. Endocrinology 154(10):3888-3899

30. Kalich-Philosoph L, Roness H, Carmely A, Fishel-Bartal $M$, Ligumsky $H$, Paglin $S$ et al (2013) Cyclophosphamide triggers follicle ac- 
tivation and "burnout;" AS101 prevents follicle loss and preserves fertility. Sci Transl Med 5(185):185ra62-185ra62

31. Pascuali N, Scotti L, Di Pietro M, Oubiña G, Bas D, May M et al (2018) Ceramide-1-phosphate has protective properties against cyclophosphamideinduced ovarian damage in a mice model of premature ovarian failure. Hum Reprod 33(5):844-859

32. Abir R, Fisch B, Jessel S, Felz C, Ben-Haroush A, Orvieto $R$ (2011) Improving posttransplantation survival of human ovarian tissue by treating the host and graft. Fertil Steril 95(4):1205-1210

33. Oktay K, Bedoschi G, Pacheco F, Turan V, Emirdar V (2016) First pregnancies, live birth, and in vitro fertilization outcomes after transplantation of frozen-banked ovarian tissue with a human extracellular matrix scaffold using robot-assisted minimally invasive surgery. Am J Obstet Gynecol 214(1):94.e1-94.e9

34. O'Brien MJ, Pendola JK, Eppig JJ (2003) A revised protocol for in vitro development of mouse oocytes from primordial follicles dramatically improves their developmental competence. Biol Reprod 68(5):1682-1686

35. Eppig JJ, O'Brien MJ (1996) Development in vitro of mouse oocytes from primordial follicles. Biol Reprod 54(1):197-207

36. Liebenthron J, Köster M, Drengner C, Reinsberg J, van der Ven H, Montag M (2013) The impact of culture conditions on early follicle recruitment and growth from human ovarian cortex biopsies in vitro. Fertil Steril 100(2):483-485

37. Winkler-Crepaz K, Nederegger V, Ayuandari S, Rosenfellner D, Zervomanolakis I, Hofer S et al (2014) Novel dynamic culture system to support initiation of primordial follicle growth in prepubertal mouse ovaries. Fertil Steril 102(3):864-870.e2

38. Telfer EE, McLaughlin M, Ding C, Thong KJ (2008) A two-step serum-free culture system supports development of human oocytes from primordial follicles in the presence of activin. Hum Reprod 23(5):1151-1158

39. Jin SY, Lei L, Shikanov A, Shea LD, Woodruff TK (2010) A novel two-step strategy for in vitro culture of early-stage ovarian follicles in the mouse. Fertil Steril 93(8):2633-2639

40. Martinez F (2017) Update on fertility preservation from the Barcelona International Society for Fertility Preservation-ESHRE-ASRM 2015 expert meeting: indications, results and future perspectives. Fertil Steril 108(3):407-411.e11

41. McLaughlin $M$, Albertini DF, Wallace WHB, Anderson RA, Telfer EE (2018) Metaphase II oocytes from human unilaminar follicles grown in a multi-step culture system. Mol Hum Reprod 24(3):135-142

42. Xiao S, Zhang J, Romero MM, Smith KN, Shea LD, Woodruff TK (2015) In vitro follicle growth supports human oocyte meiotic maturation. Sci Rep 5(1):17323-17325

43. Hsueh AJW, Kawamura K, Cheng Y, Fauser BCJM (2015) Intraovarian control of early folliculogenesis. Endocr Rev 36(1):1-24

44. Ayuandari S, Winkler-Crepaz K, Paulitsch M, Wagner C, Zavadil C, Manzl C et al (2016) Follicular growth after xenotransplantation of cryopreserved/thawed human ovarian tissue in SCID mice: dynamics and molecular aspects. J Assist Reprod Genet 33(12):1585-1593

45. Kawamura K, Cheng Y, Suzuki N, Deguchi M, Sato $Y$, Takae S et al (2013) Hippo signaling disruption and Akt stimulation of ovarian follicles for infertility treatment. Proc Natl Acad Sci USA 110(43):17474-17479

46. Kawamura K, Cheng Y,Sun Y-P, Zhai J,Diaz-GarciaC, Simon $C$ et al (2015) Ovary transplantation: to activate or not to activate. Hum Reprod 30(11):2457-2460

47. Suzuki N, Yoshioka N, Takae S, Sugishita $Y$, Tamura M, Hashimoto S et al (2015) Successful fertility preservation following ovarian tissue vitrification in patients with primary ovarian insufficiency. Hum Reprod 30(3):608-615

48. Spears N, Lopes F, Stefansdottir A, Rossi V, De Felici M, Anderson RA et al (2019) Ovarian damage from chemotherapy and current approaches to its protection. Hum Reprod Update 25(6):673-693

49. Laronda MM, Jakus AE, Whelan KA, Wertheim JA, Shah RN, Woodruff TK (2015) Initiation of puberty in mice following decellularized ovary transplant. Biomaterials 50:20-29

50. Shea LD, Woodruff TK, Shikanov A (2014) Bioengineering the ovarian follicle microenvironment. Annu Rev Biomed Eng 16(1):29-52

51. Amorim CA, Shikanov A (2016) The artificial ovary: current status and future perspectives. Future Oncol 12(20):2323-2332

52. Soares M, Sahrari K, Amorim CA, Saussoy $P$, Donnez J, Dolmans M-M (2015) Evaluation of a human ovarian follicle isolation technique to obtain disease-free follicle suspensions before safely grafting to cancer patients. Fertil Steril 104(3):672-672

53. Morohaku K, Tanimoto R, Sasaki K, KawaharaMiki R, Kono T, Hayashi K et al (2016) Complete in vitro generation of fertile oocytes from mouse primordial germ cells. Proc Natl Acad Sci USA 113(32):9021-9026

54. Truman AM, Tilly JL, Woods DC (2017) Ovarian regeneration: The potential for stem cell contribution in the postnatal ovary to sustained endocrine function. Mol Cell Endocrinol 445:74-84

55. Donnez J, Squifflet J, Pirard C, Demylle D, Delbaere A, Armenio L et al (2011) Live birth after allografting of ovarian cortex between genetically non-identical sisters. Hum Reprod 26(6):1384-1388

56. Dittrich R, Lotz L, Fehm T, Krüssel J, von Wolff M, Toth B et al (2015) Xenotransplantation of cryopreserved human ovarian tissue-a systematic review of MII oocyte maturation and discussion of it as a realistic option for restoring fertility after cancer treatment. Fertil Steril 103(6):1557-1565

57. Tschudin S, Bitzer J (2009) Psychological aspects of fertility preservation in men and women affected by cancer and other life-threatening diseases. Hum Reprod Update 15(5):587-597

58. Böttcher B, Paul N (2013) Personale Autonomie: Diskussion eines zentralen ethischen Konzepts am Beispiel von fertilitätsprotektiven Maßnahmen be Krebspatientinnen. EthikMed 25:47-54

59. Böttcher B, Goeckenjan M (2013) Jetzt oder nie - ethische Aspekte der Fertilitätsprotektion bei onkologischen Patientinnen. Gynäkologe 46(9):37-41

Hinweis des Verlags. Der Verlag bleibt in Hinblick auf geografische Zuordnungen und Gebietsbezeichnungen in veröffentlichten Karten und Institutsadressen neutral.

\section{PflegeDossier Cannabinoide}

\author{
Pflege Dossier \\ zertifizierte Fortbilduns
}

Cannabinoide in der Geriatrie, Onkologie, Schmerztherapie und Palliation

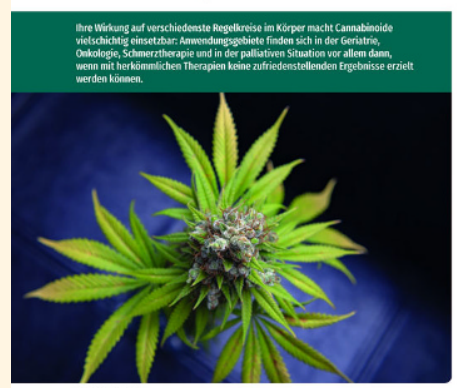

PROCARE SpringerPflege

Cannabinoide wirken neuroprotektiv, schmerzstillend und entzündungshemmend und sie zeigen auch verhaltensbezogene positive Effekte. Ein äußerst vielseitiges Cannabinoid ist Dronabinol, das seit 2004 in Österreich als Rezepturarzneimittel verfügbar ist. Seine Einsatzmöglichkeiten sind daher vielschichtig, wobei Dronabinol in der Geriatrie, Onkologie, Schmerzbehandlung und Palliativbetreuung gerne als zusätzliche Therapiemaßnahme genützt wird. Durch ihre große Nähe zum Patienten kommt Diplomierten Gesundheits- und Krankenpflegepersonen auch in diesem Zusammenhang eine wichtige Rolle zu.

Zur Fortbildung für die Gesundheits- und Krankenpflege zum Thema Cannabinoide steht ab sofort ein PflegeDossier von PROCARE/SpringerPflege zur Verfügung, das bei Springer Wien bestellt werden kann: procare@springer.at

\section{Aus dem Internet herunterladen}

Die Fortbildung ist auch online unter www.springermedizin.at/pflegedossier downloadbar, sie ist vom Österreichischen Gesundheits- und Krankenpflegeverband (ÖGKV) zertifiziert und mit 3 Pflegefortbildungspunkten - PFP ${ }^{\circledR}$ bewertet. Die Teilnahme ist durch Unterstützung der Firma $\mathrm{C}^{3}$ Ethics Austria GmbH kostenlos. 
Hier steht eine Anzeige.

\section{曾 Springer}

